\title{
Effects of the attractive interactions in the thermodynamic, dynamic, and structural anomalies of a two length scale potential
}

Cite as: J. Chem. Phys. 133, 244506 (2010); https://doi.org/10.1063/1.3511704

Submitted: 31 May 2010. Accepted: 17 October 2010 . Published Online: 29 December 2010

Jonathas Nunes da Silva, Evy Salcedo, Alan Barros de Oliveira, and Marcia C. Barbosa

\section{ARTICLES YOU MAY BE INTERESTED IN}

Thermodynamic, dynamic, and structural anomalies for shoulderlike potentials

The Journal of Chemical Physics 131, 094504 (2009); https://doi.org/10.1063/1.3213615

Comparison of liquid-state anomalies in Stillinger-Weber models of water, silicon, and germanium

The Journal of Chemical Physics 145, 214502 (2016); https://doi.org/10.1063/1.4967939

Comment on "The putative liquid-liquid transition is a liquid-solid transition in atomistic models of water" [I and II: J. Chem. Phys. 135, 134503 (2011); J. Chem. Phys. 138, 214504 (2013)]

The Journal of Chemical Physics 148, 137101 (2018); https://doi.org/10.1063/1.5029463

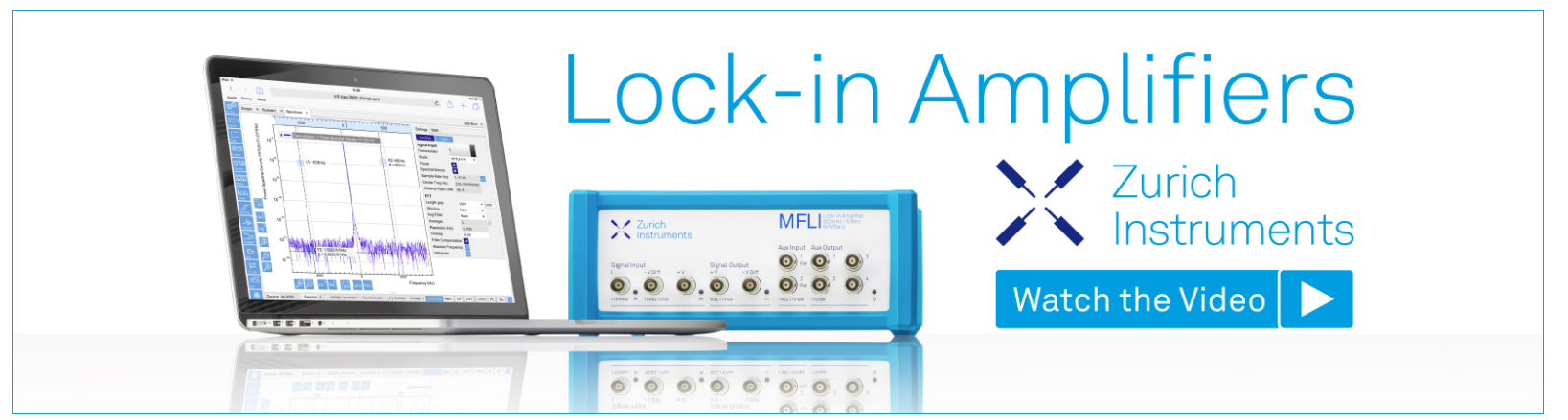

J. Chem. Phys. 133, 244506 (2010); https://doi.org/10.1063/1.3511704 


\title{
Effects of the attractive interactions in the thermodynamic, dynamic, and structural anomalies of a two length scale potential
}

\author{
Jonathas Nunes da Silva, ${ }^{1, a)}$ Evy Salcedo, ${ }^{2, b)}$ Alan Barros de Oliveira, ${ }^{3, c)}$ \\ and Marcia C. Barbosa ${ }^{1}$ \\ ${ }^{1}$ Instituto de Física, Universidade Federal do Rio Grande do Sul, Caixa Postal 15051, 91501-970, \\ Porto Alegre, RS, Brazil \\ ${ }^{2}$ Departamento de Física, Universidade Federal de Santa Catarina, Florianópolis, SC, 88010-970, Brazil \\ ${ }^{3}$ Departamento de Física, Universidade Federal de Ouro Preto, Ouro Preto, MG, 35400-000, Brazil
}

(Received 31 May 2010; accepted 17 October 2010; published online 29 December 2010)

\begin{abstract}
Using molecular dynamic simulations, we study a system of particles interacting through a continuous core-softened potentials consisting of a hard core, a shoulder at closest distances, and an attractive well at further distance. We obtain the pressure-temperature phase diagram of this system for various depths of the tunable attractive well. Since this is a two length scale potential, density, diffusion, and structural anomalies are expected. We show that the effect of increasing the attractive interaction between the molecules is to shrink the region in pressure in which the density and the diffusion anomalies are present. If the attractive forces are too strong, particle will be predominantly in one of the two length scales and no density of diffusion anomaly is observed. The structural anomalous region is present for all the cases. (C) 2010 American Institute of Physics. [doi:10.1063/1.3511704]
\end{abstract}

\section{INTRODUCTION}

Water is one of the most abundant substance on the planet, however, its thermodynamic and dynamic properties are away from being fully understood. ${ }^{1}$ Unlike other liquids, its specific volume at ambient pressure increases when cooled below $T=4{ }^{\circ} \mathrm{C} .{ }^{2}$ Besides, the isothermal compressibility, $\kappa_{T}$ and the specific heat at constant pressure, $C_{P}$, have a minimum at $T=T_{\min }$. For temperatures below $T_{\min }, \kappa_{T}^{3,4}$ and $C_{P}$ increase with temperature decrease and above $T_{\min },{ }^{2,5} \kappa_{T}$ and $C_{P}$ increase with temperature increase.

In the last years, the interest for the supercooled region of the pressure-temperature phase diagram has increased. In this region, water is forced to be in liquid state due to fast freezing of the system. Different from normal liquids, the self-diffusion, $D$, of the supercooled water increases with the compression up to maximum value $D_{\max }(T)$ at $p=p_{D_{\max }} \cdot{ }^{3,5}$ Beyond this maximum value, for higher pressures, the "normal" behaviors are restored and diffusion decreases with pressure. $^{6-10}$ These results are supported by numerical simulation using the SPC/E water model where the supercooled region is easily accessed. ${ }^{11}$

In addition to the thermodynamic and dynamic anomalies, water also exhibits a very complex phase diagram with a large number of stable solid phases and two amorphous phases, the high density amorphous phase and low density amorphous phase. ${ }^{12,13}$ Supported by numerical results, it is speculated that the two amorphous phases give rise to two liquid phases in the deeply supercooled region: the high density liquid and low density liquid. ${ }^{14,15}$ A possible scenario is that the transition line between these two liquid phases finishes

\footnotetext{
${ }^{a)}$ Author to whom correspondence should be addressed. Electronic mail: jonathas@if.ufrgs.br.

b) Electronic mail: esalcedo@fsc.ufsc.br.

c)Electronic mail: oliveira@iceb.ufop.br.
}

at a liquid-liquid critical point. ${ }^{16}$ The presence of a critical point would also explain the increase in the isothermal compressibility and other response functions.

Water is not an isolated case. Thermodynamic anomalies do not occur only in water, experiments for $\mathrm{Te},{ }^{17} \mathrm{Ga}$, $\mathrm{Bi}, \mathrm{S},{ }^{18,19}$ and $\mathrm{Ge}_{15} \mathrm{Te}_{85},{ }^{20}$ liquid metals ${ }^{21}$ and graphite ${ }^{22}$ and simulations for silica, ${ }^{23-25}$ silicon, ${ }^{26}$ and $\mathrm{BeF}_{2}{ }^{23}$ shown that these systems also have thermodynamic anomalies. In addition, silica ${ }^{25,27}$ and silicon ${ }^{28}$ show diffusion anomalous behavior. Unfortunately a coherent and general interpretation of the mechanism, which leads to the anomalies and to the two liquid phases, is still missing.

In order to understand about the fundamental origin of the anomalous behaviors and multiple liquid phases, simplified isotropic pair interaction potentials were developed. They are capable to reproduce qualitatively the properties observed by complex anisotropic potentials. These simplified potentials are called core-softened (CS) potential. The CS potential is formed by repulsive core with a softening region as a shoulder or a ramp. ${ }^{29-40}$ This approach generates models analytically ${ }^{41-44}$ and computationally ${ }^{29-37}$ tractable. Most of these CS potential exhibit thermodynamic and dynamic anomalies and show the presence of two liquid phases.

In addition, these systems also present a number of solid phases, ${ }^{37,45-48}$ including low density solid phases and reentrant melting lines. The existence of anomalous properties, two liquid phases, and polymorphism opened a discussion about the relation between these features and the form of the potential.

Following the idea of simplified models to explain such properties, de Oliveira et al. ${ }^{35,49-53}$ proposed a simple CS model. It has a repulsive core that exhibits a region of softening where the slope changes drastically. This model exhibits density, diffusion, and structural anomalies like the 
anomalies present in experiments ${ }^{3,5}$ and simulations ${ }^{6-8}$ for water. This simple system has no attraction between the particles and, therefore, no liquid-gas or liquid-liquid critical points are present. Realistic models for representing the intermolecular forces should have attractive interactions since most molecules attract each other either due to van der Waals interactions or to more sophisticated electrostatic forces. If instead the CS model represents effective interaction between group of particles, ${ }^{54,55}$ the attractive interaction would be the ingredient for the appearance of two liquid phases.

Which effect in the pressure-temperature phase diagram one might expect from the addition of a larger attractive part in the potential? For one length scale potentials, the increase of the attractive well leads to an increase in the temperature of the liquid-gas critical point. In the case of the continuous two length scale potential, the same behavior might be expected for the liquid-gas critical point but it is not clear which effect the depth of the well has in the location in the pressuretemperature phase diagram of the liquid-liquid critical point. Moreover, it is also not obvious which effect the attraction has in the location in the pressure-temperature phase diagram of the density, diffusion, and structural anomalous regions.

In this paper, we address these two questions by studying the pressure-temperature phase diagram of a potential with a repulsive core followed by a tunable attractive well. We check if the introduction of the attraction between particles affects the liquid-liquid critical point and the density, diffusion, and structural anomalies.

The remaining of this paper goes as follows. In Sec. II, the model is introduced and the methods are presented. Details of simulations are given in Sec. III. In Sec. IV, the results are discussed and, finally, the conclusion are made in Sec. V.

\section{THE MODEL}

The model consists of a system of $N$ particles of diameter $\sigma$, inside a cubic box with volume $V$, resulting in a number density $\rho=N / V$. The effective interacting potential between particles is given by

$$
\begin{aligned}
U^{*}(r)= & 4\left[\left(\frac{\sigma}{r}\right)^{12}-\left(\frac{\sigma}{r}\right)^{6}\right]+a \exp \left[-\frac{1}{c^{2}}\left(\frac{r-r_{0}}{\sigma}\right)^{2}\right] \\
& +b \exp \left[-\frac{1}{d^{2}}\left(\frac{r-r_{1}}{\sigma}\right)^{2}\right]
\end{aligned}
$$

where $U^{*}(r)=U(r) / \varepsilon$. The first term of Eq. (1) is a LennardJones potential of well depth $\varepsilon$. The second and third terms are Gaussians centered on radius $r=r_{0}$ and $r=r_{1}$, with heights $a$ and $b$, and widths $c$ and $d$, respectively. This potential can represent a whole family of two length scale intermolecular interactions, from a deep double wells potential ${ }^{5,57}$ to a repulsive shoulder, ${ }^{33}$ depending on the choice of the values of the parameters.

For $b=0$, the attractive part vanishes and the potential becomes purely repulsive. This case was previously studied for determining the pressure-temperature phase diagram as well as the regions where waterlike anomalies occur. ${ }^{35,49}$

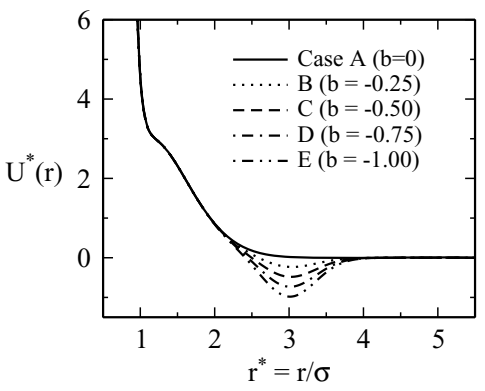

FIG. 1. Interaction potential Eq. (1) with parameters $a=5.0, r_{0} / \sigma=0.7$, $c=1.0, r_{1} / \sigma=3.0$, and $d=0.5$ for all cases. $b$ is shown in Table I for each case.

How the addition of an attractive part in the potential affects the overall pressure-temperature phase diagram? In order to answer this question in Secs. III-V, we obtain the pressure-temperature phase diagram of the potentials illustrated in Fig. 1 where the attractive part is increased systematically without changing the core-softened part of the potential. This is done by fixing the parameters of Eq. (1): $a=5.0$, $r_{0} / \sigma=0.7, c=1.0, r_{1} / \sigma=3.0, d=0.5$ and varying the parameter $b$ as shown in Table I for the five cases studied in this work.

\section{DETAILS OF SIMULATIONS}

For the case in which $b=0$, the results shown in this paper were adapted from Refs. 35 and 49. For the other cases $(b \neq 0)$ the details of the simulations go as follows.

The quantities of interest were obtained by NVT-constant molecular dynamics using the LAMMPS package. ${ }^{58}$ The system consists of $N=1372$ particles into a cubic box with periodic boundary conditions in all directions. The interaction potential between particles, Eq. (1), has a cutoff of $r_{c}=4.5 \sigma$ and the potential was shifted in order to have $U=0$ at $r_{c}$. The Nose-Hoover heat-bath with coupling parameter $Q=2$ was used in order to keep fixed the temperature.

All simulations were initialized in a liquid phase previously equilibrated over $5 \times 10^{5}$ steps at $T^{*}=0.6$. The time step used was 0.001 in reduced units and the runs were carried out for a total of $3 \times 10^{6}$ steps, dumping instantaneous configurations for every 2000 steps, giving then a total of 1500 independent configurations. The first 200 configurations were discarded for equilibration purposes, thus 1300 configurations were used for sampling averages. The highly number of independent configurations guarantees percentage uncertainty of

TABLE I. Parameter $b$ in the potential Eq. (1) for each case studied in this work. The other parameters are $a=5.0, r_{0} / \sigma=0.7, c=1.0, r_{1} / \sigma=3.0$, and $d=0.5$ for the five cases.

\begin{tabular}{lc}
\hline \hline & $b$ \\
\hline Case A & 0 \\
Case B & -0.25 \\
Case C & -0.50 \\
Case D & -0.75 \\
Case E & -1.00 \\
\hline \hline
\end{tabular}


pressure and temperature of the isochores on PT diagram to be smaller than $1 \%$. Thus, the uncertainties are smaller than the point size on PT diagram.

Preliminary simulations showed that depending on the chosen temperature and density, the system was in a fluid phase but became metastable with respect to the solid phase. In order to locate the phase boundary between the solid and the fluid phases, two sets of simulations were carried out, one beginning with the molecules in an ordered crystal structure and the other beginning with molecules in a random liquid state, obtained from previous equilibrated simulations. The stability of the system was checked by analyzing the dependence of pressure on density and also by visual analysis of the final structure, searching for cavitation.

The critical points were located as the locus where the isochores cross. The coexistence line between two different phases was estimated by the mean point between the spinodals.

Temperature, pressure, density, and diffusion are measured in dimensionless units,

$$
\begin{aligned}
T^{*} & \equiv \frac{k_{B} T}{\epsilon}, \\
\rho^{*} & \equiv \rho \sigma^{3}, \\
P^{*} & \equiv \frac{P \sigma^{3}}{\epsilon}, \\
D^{*} & \equiv \frac{D(m / \epsilon)^{1 / 2}}{\sigma} .
\end{aligned}
$$

The pressure of the system is calculated by means of the the virial theorem,

$$
P=\rho k_{B} T+\frac{1}{3 V}\left\langle\sum_{i<j} \mathbf{f}\left(\mathbf{r}_{i j}\right) \cdot \mathbf{r}_{i j}\right\rangle,
$$

where $\mathbf{r}_{i j}$ is the vector that connects particle $i$ with particle $j$, $\mathbf{f}(\mathbf{r})=-\boldsymbol{\nabla} \mathbf{U}(\mathbf{r})$. The symbol $\langle\ldots\rangle$ indicates ensemble average.

The mobility of particles is evaluated by the mean square displacement, given by

$$
\left\langle\Delta r(\tau)^{2}\right\rangle=\left\langle\left[r\left(\tau_{0}+\tau\right)-r\left(\tau_{0}\right)\right]^{2}\right\rangle .
$$

The diffusion coefficient is then obtained from Einstein's relation, namely

$$
D=\lim _{\tau \rightarrow \infty} \frac{\left\langle\Delta r(\tau)^{2}\right\rangle}{6 \tau} .
$$

For normal fluids, the diffusion at constant temperature grows with decreasing density. Actually in most cases it is expected that it would follow the Stokes-Einstein relation, i.e., $D \propto T$.

The structure of the system was analyzed by using the translational order parameter, defined as $8,25,59,60$

$$
t=\int_{0}^{\xi_{c}}|g(\xi)-1| d \xi,
$$

where $\xi=r \rho^{1 / 3}$ is the inter-particle distance divided by the average separation between pairs of particles $\rho^{-1 / 3}$. Here $g(\xi)$ is the distribution function of pairs. $\xi_{c}$ is the distance cutoff, where we use half of the length of the simulation box, $r_{c}$, multiplied by $\rho^{1 / 3}$. Another alternative to $r_{c}$ would be the first or the second peak in the $g(r)$. Our choice is preferable, first, because it is the maximum distance allowed for the calculation of $g(r)^{61}$ giving us a better approach allowed for $t$. Second, the peaks of $g(r)$ change place according to density and temperature of the system. Thus additional work would be necessary to find such positions.

For the ideal gas, $g=1$ thus $t=0$. As the system becomes more structured a long range order $(g \neq 1)$ appears and $t$ assumes large values. The translational order parameter has its maximum value in the crystal phase. Therefore, $t$ gives a measurement of how close is the fluid close to the crystallization. For a fixed temperature normal fluids present a monotonic $t(\rho)$ curve, increasing with density.

\section{RESULTS}

In this section we show what is the effect of increasing the attractive part of the potential in the pressure-temperature phase diagram regarding: (a) the presence and location of different phases and critical points; (b) the presence of a density anomalous region; (c) the presence of diffusion anomalous region; (d) the presence of a structural anomalous region.

\section{A. Phase diagram}

Figures 2(a)-2(e) illustrates the pressure-temperature phase diagram obtained through simulations for the cases A-E using the potential shown in Fig. 1. The gray lines are the isochores. In all cases, at high temperatures the system exhibit a fluid phase and a gas phase. These two phases coexist at a first order line that ends at a critical point (open circle at figure) for B-E cases. At low temperatures and high pressures there are two liquid phases coexisting at a first order line (not shown) ending at a second critical point (filled point at figure) for $\mathrm{C}-\mathrm{E}$ cases. These critical points are identified in the graph by the region where isochores cross. The location was also checked by the peak of the specific heat. The coexistence line between the gas and the liquid phases and between the low density liquid phase and the high density liquid phase (both illustrated as solid lines) were obtained as the mean point between the respective spinodals.

At low pressures and temperatures, the region where no isochore is present the liquid phase is metastable or unstable against solid phases. Since polymorphism characterizes the CS potentials, a number of solid phases might be expected. Here we do not explore the stability of the different solids. Also, in the case E, the liquid-liquid phase transition appears at negative pressures. The negative pressure indicates that the system wants to contract but since the volume is fixed it is not possible.

The main effect of the increase of depth of the attractive well at the location of the different phases in the $P T$ phase diagram is to move the critical points to higher temperature. To get some intuitive understanding of this effect at liquidgas critical point, we use the cluster expansion methods for 

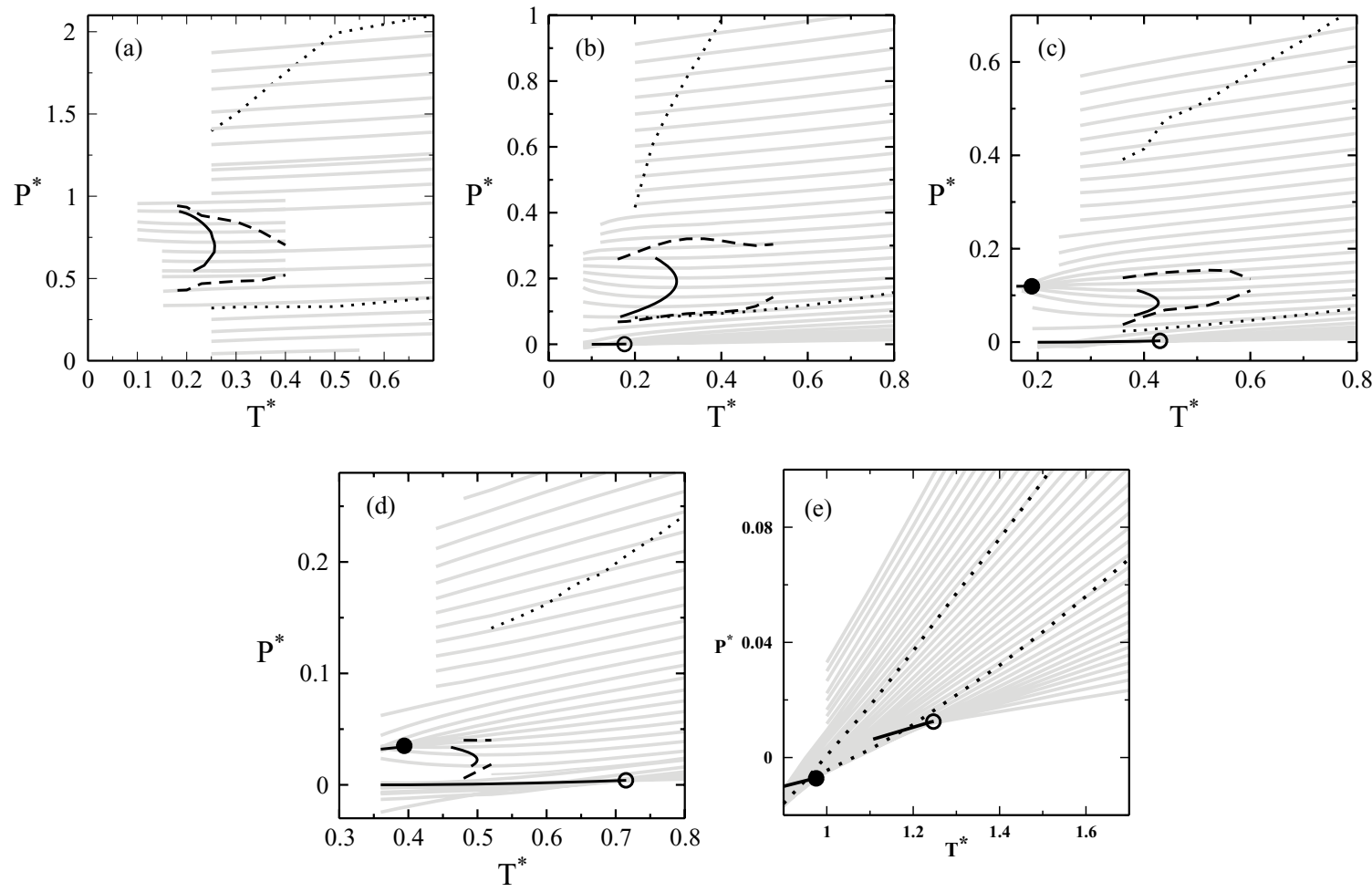

FIG. 2. Pressure-temperature phase diagram. The gray lines are the isochores. (a) Case A $(b=0): \rho^{*}=0.04,0.06,0.07, \ldots$, and 0.20 from bottom to top. (b) Case $\mathrm{B}(b=-0.25): \rho^{*}=0.01,0.015, \ldots$, and 0.165 from bottom to top. (c) Case $\mathrm{C}(b=-0.50)$ : same as panel (b), (d) case $\mathrm{D}(b=-0.75)$ : same as panel (b). (e) Case $\mathrm{E}(b=-1.00): \rho^{*}=0.02,0.025, \ldots$, and 0.2 from bottom to top. The solid, bold line is the $T M D$ line, the dashed line marks the diffusion extrema and the dotted line bounds the region of structural anomaly. The filled and open circles are the liquid-liquid and liquid-gas critical points, respectively. The thin solid lines are the coexistence lines.

low density:

$$
\begin{aligned}
\frac{\beta P}{\rho}= & 1-2 \pi \rho \int f(r) r^{2} d r-\frac{8 \pi^{2} \rho^{2}}{3} \\
& \iiint f(r) f\left(r^{\prime}\right) f\left(\left|r-r^{\prime}\right|\right) \sin \theta r^{2} r^{\prime 2} d r d r^{\prime} d \theta,
\end{aligned}
$$

where $f(r)=e^{-\beta U(r)}-1$. This method allows to approach to $P \rho$ phase diagram from pair interaction potential $U(r)$. At $P \rho$ phase diagram, critical points are located as

$$
\begin{gathered}
\frac{\partial P}{\partial \rho}=0, \\
\frac{\partial^{2} P}{\partial \rho^{2}}=0 .
\end{gathered}
$$

The low density behavior obtained using the cluster expansion is illustrated in Fig. 3. For $T^{*}=0.60$, Fig. 3 shows the pressure-density phase diagram for $b$ $=0.0,-0.25,-0.50,-0.75,-1.00$ using the second and the third virial. For $b=-1.00$ the unstable region of the pressure-density phase diagram is large and the system at this temperature is deep in the liquid-gas coexistence region of the pressure-temperature phase diagram. For $b=-0.75$ the unstable region is present but is rather small. For $b$ $=0.0,-0.25$, and -0.50 no unstable region in the pressuredensity phase diagram is observed indicating that the system is above the liquid-gas transition and that $T=0.60$ is larger than the critical point temperature. The comparison between the cases with $b=0.0,-0.25$, and -0.50 suggests that since the slope of the pressure-density phase diagram increases as $b$ increases, the liquid-gas critical temperature decreases as $b$ increases, $T_{c}^{*}(b=-0.25)<T_{c}^{*}(b=-0.50)$ $<T_{c}^{*}(b=-0.75)<T_{c}^{*}(b=-1.00)$. Consequently, the attractive part favors the liquid phase to exist for higher temperatures what is also observed in discontinuous potentials. ${ }^{62,63}$ Figure 4 obtained from the simulations summarizes the effect of the attractive part in the location of the critical points in the pressure-temperature diagram.

At high densities where the liquid-liquid phase transition is present, the cluster expansion with second and third

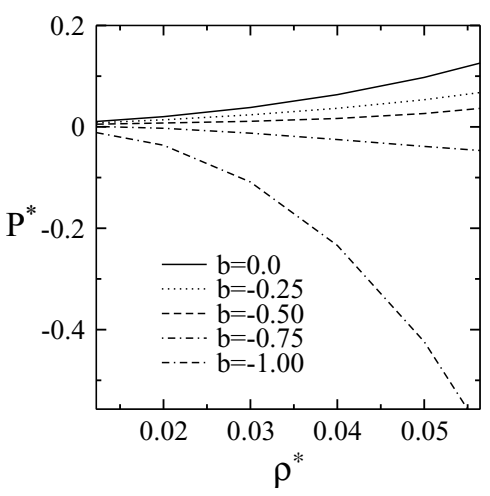

FIG. 3. Pressure vs density obtained using virial expansion for $T^{*}=0.60$ for the cases $b=0.0,-0.25,-0.5,-0.75$, and -1.00 from top to bottom. 


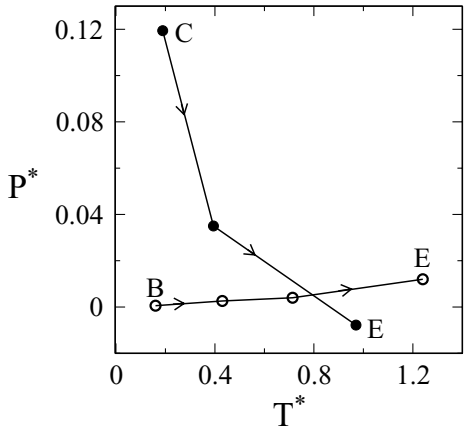

FIG. 4. Location of the critical points obtained using NVT simulations for the cases B-E considered in this work. The case A does not present any fluidfluid critical point whereas the case B has a liquid-gas but no liquid-liquid critical point. The critical points were located by the crossing of the isochores. The symbols have the same meaning as in Figs. 2(a)-2(e), i.e., filled and open circles mark the liquid-liquid and liquid-gas critical points, respectively. The arrows indicate the direction of increasing the attractive interaction.

virial is not appropriated. Simulations show that as $b$ decreases the pressure needed to form the high density liquid phase, decreases. The attractive part favors the high density liquid phase over the low density liquid phase. The attraction leads in this case to a more compact liquid phase what is also observed in discontinuous potentials. ${ }^{62,63}$

\section{B. Density anomaly}

In order to test for the presence of density anomaly, we proceed as follows. From the Maxwell relation,

$$
\left(\frac{\partial V}{\partial T}\right)_{P}=-\left(\frac{\partial P}{\partial T}\right)_{V}\left(\frac{\partial V}{\partial P}\right)_{T},
$$

the maximum in $\rho(T)$ versus temperature at constant pressure given by $(\partial \rho / \partial T)_{P}=0$ is equivalent to the minimum of the pressure versus temperature at constant density, namely $(\partial P / \partial T)_{\rho}=0$. While the former is suitable for $N P T$-constant experiments/simulations the latter is more convenient for our NVT-ensemble study, thus adopted in this work.

The temperatures of minimum pressure at constant density or equivalently the temperatures of maximum density at constant pressure, the TMD, obtained by $N V T$ simulations are illustrated in Figs. 2(a)-2(d) as a bold line. As the attractive well becomes deeper, the region in the pressuretemperature phase diagram occupied by the density anomalous region shrinks and moves to lower pressures and higher temperatures until to the limiting case, $b=-1.00$, shown in Fig. 2(e), in which no density anomaly is present. This result can be understood using the radial distribution function. The $T M D$ is related to the presence of large regions in the system in which particles are in two preferential distances represented by the first scale and the second scale represented by the two first peaks in the radial distribution function in our potential. $^{40,64-66}$

Figure 5(e) illustrates the behavior expected for normal liquids. As the temperature is increased the percentage of particles in closest scales decreases. The decrease of particles in the first scale leads to a decrease of density with the increase of temperature. In Figs. 5(a)-5(d), as the temperature is in-
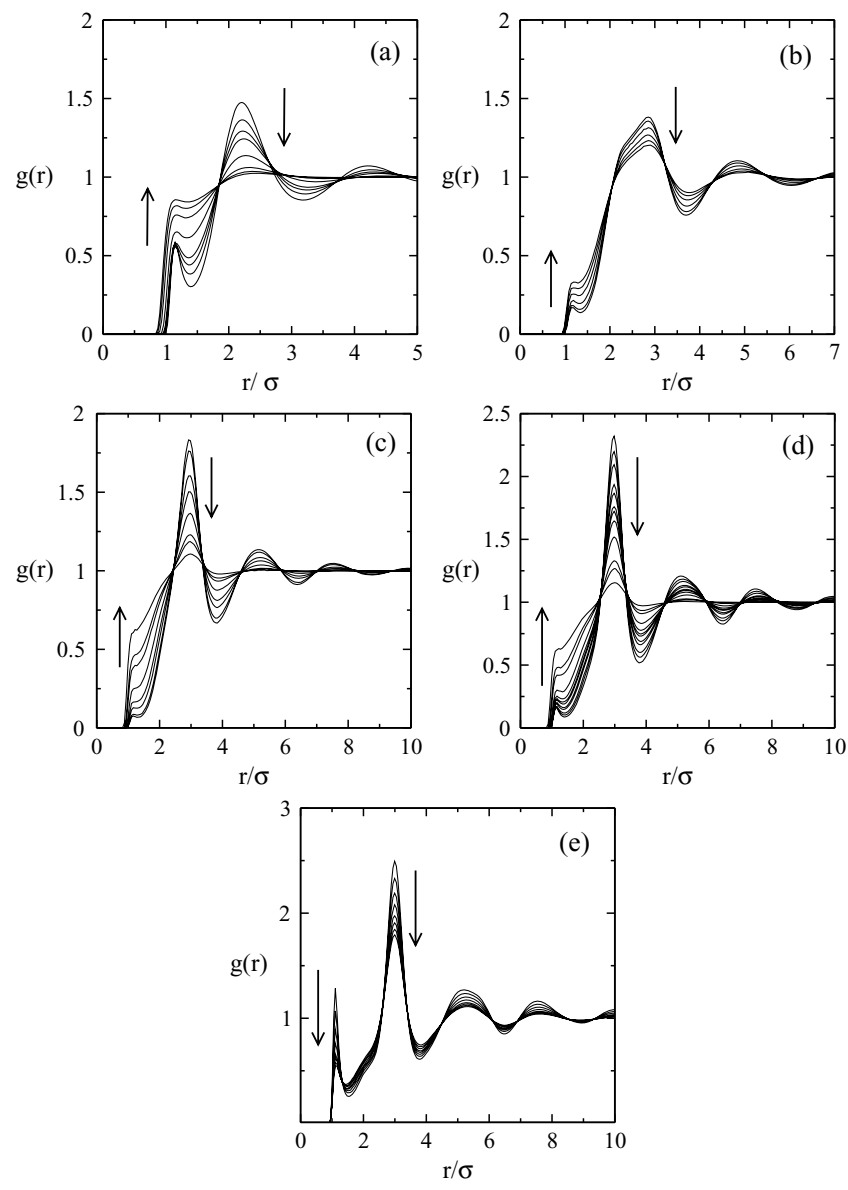

FIG. 5. Radial distribution function obtained using $N V T$ simulations vs distance for (a) case A $(b=0.0)$ with $\rho^{*}=0.14$ and $T^{*}=0.25,0.35,0.45,0.55,1.0,2.0,3.0$, and 4.0 ; (b) case B $(b=-0.25)$ with $\rho^{*}=0.085$ and $T^{*}=0.32,0.36,0.44,0.56,0.68$, and 0.80 ; (c) case C $(b=-0.50)$ with $\rho^{*}=0.06$ and $T^{*}=0.44,0.48,0.60,0.72,1.0,1.6$, 2.0, and 3.5; (d) case $\mathrm{D}(b=-0.75)$ with $\rho^{*}=0.06$ and $T^{*}=0.40,0.44$, $0.48,0.56,0.60,0.68,0.72,0.80$, and 1.0 ; and (e) case $\mathrm{E}(b=-1.00)$ with $\rho^{*}=0.07$ and $T^{*}=0.45,0.50, \ldots$, and 0.80 . The arrows indicate the direction of increasing temperature.

creased the percentage of particles at the closest distance increases while the percentage of particles in the second scale decreases. The increase of particles in the first scale leads to an increase of density with temperature what characterizes the anomalous region. The density anomaly is, therefore, related to the increase of the probability of particles to be in the first scale when the temperature is increased while the percentage of particles in the second scale decreases. As the potential becomes highly attractive this "mobility" between scales disappears, i.e., the high density liquid becomes dominant and no anomalous region is observed.

\section{Diffusion anomaly}

The mobility was obtained from the slope of the mean square displacement as shown in Eqs. (4) and (5). Figures 6(a)-6(e) show the behavior of the dimensionless translational diffusion coefficient, $D^{*}$, as the function of the dimensionless density, $\rho^{*}$, at constant temperature for $b=0.0$, $-0.25,-0.50,-0.75$, and $b=-1.00$ from the simulations. 

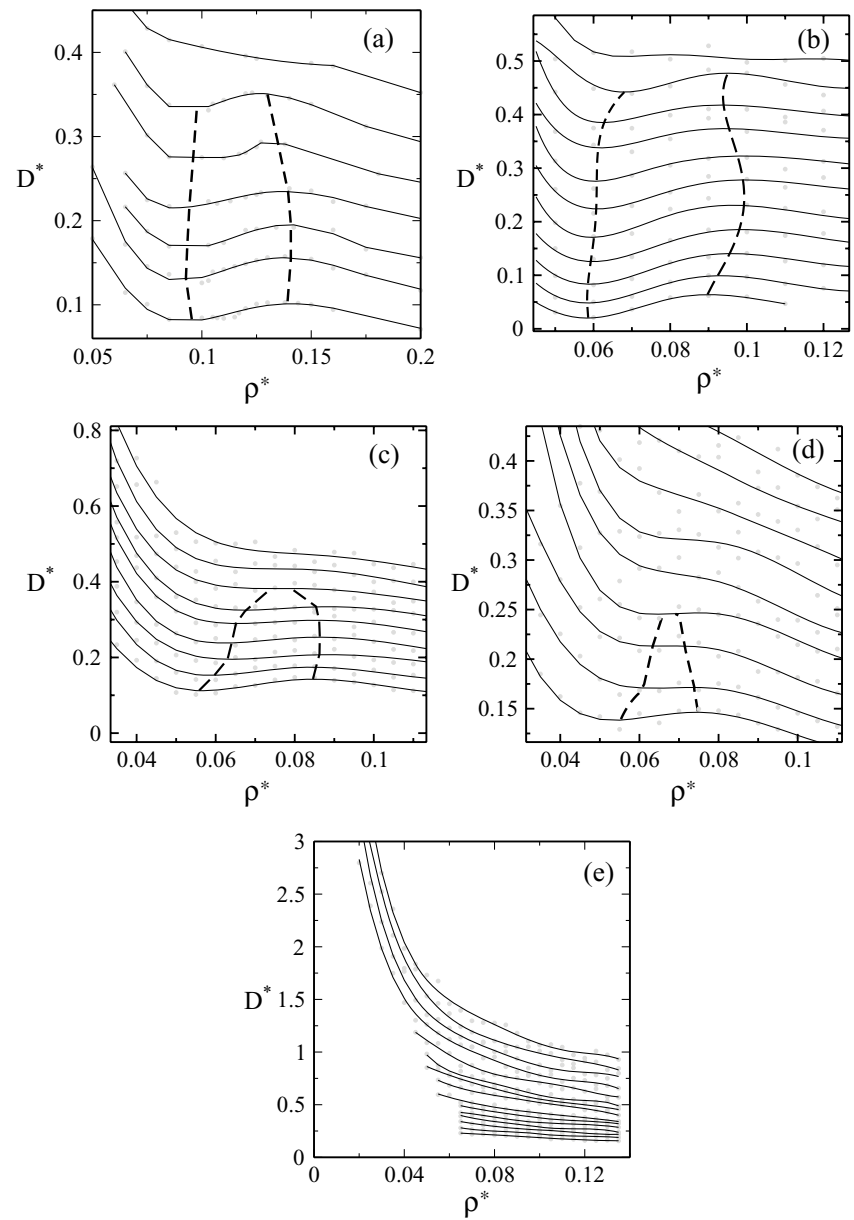

FIG. 6. The diffusion coefficient against density for the (a) case A, with isotherms $0.2,0.23,0.262,0.3,0.35,0.4$, and 0.45 from bottom to top. (b) Case B with isotherms $0.16,0.20, \ldots$, and 0.56 , (c) case $\mathrm{C}$, whose temperatures shown are $0.36,0.40, \ldots$, and 0.68 , (d) case $\mathrm{D}$, with temperatures $0.48,0.52, \ldots$, and 0.80 , and (e) case $\mathrm{E}$ with isotherms $0.70,0.75, \ldots, 1.0$, $1.10, \ldots$, and 1.70 . The dashed lines mark the local maxima/minima in the $D(\rho)$ curves. For the region enclosed by these lines particles move faster under compression. The dashed lines in this figure have the same meaning as those ones in Figs. 2(a)-2(e). The diffusion coefficient was obtained from the slope of the mean square displacement versus time. The mean square displacement was obtained by $N V T$ simulations.

The solid lines are polynomial fits to the data obtained through simulation [dots in Figs. 4(a)-4(e)].

For normal liquids, the diffusion coefficient at constant temperature decreases with increase of the density. For the cases A-D [shown in Figs. 4(a)-4(d)] $D^{*}$ anomalously increases with the increase in the density in a certain range of pressures and temperatures. From Figs. 4(a)-4(d) show that for very small and very high densities $D^{*}$ decreases with increasing density as expected for a normal liquid. For intermediate values of density, $\rho_{D \max }>\rho>\rho_{D \min }, D^{*}$ increases with increasing density what leads to local maxima at $\rho_{D \max }$ and a local minima at $\rho_{D \min }$. These local extrema in the diffusion versus density plots bound the region inside which the diffusion behaves anomalously [dashed lines in Figs. 4(a)-4(d)]. This region is mapped into the pressuretemperature diagram illustrated in Figs. 2(a)-2(d) as dashed lines in (a)-(d). As the attractive well becomes deeper, the diffusion anomalous region in the pressure-temperature phase
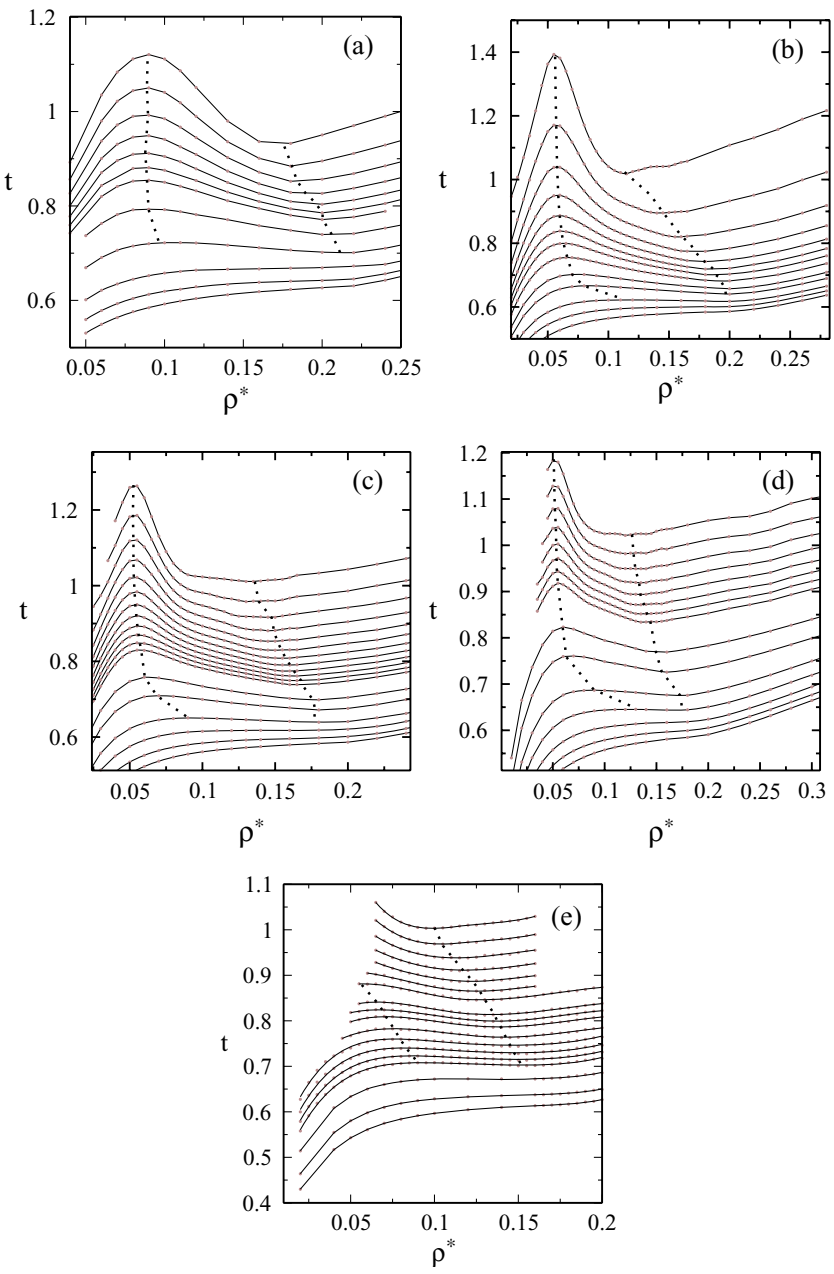

FIG. 7. Translational order parameter obtained by $N V T$ simulations against density for (a) case A, where each line correspond to an isotherm. The isotherms are: $0.25,0.30, \ldots, 0.55,0.7,1.0,1.5,2.0$, and 2.5 from top to bottom. (b) Case B, with isotherms $0.20,0.28, \ldots, 0.68,0.80,1.0,1.2,1.6$, 2.0, and 2.5 from top to bottom. (c) Case $\mathrm{C}$ whose temperatures are 0.36, $0.40, \ldots, 0.80,1.0,1.2,1.6,2.0,2.5$, and 3.0 from top to bottom. (d) Case D with $T^{*}=0.52,0.56, \ldots, 0.80,1.0,1.2,1.6,2.0,2.5,3.0$, and 3.5 from top to bottom. Finally, (e) case E with $T^{*}=0.70,0.75, \ldots, 1.0,1.10, \ldots$, $1.70,2.0,2.5$, and 3.0 from top to bottom. The dotted lines bound the region of structural anomalies, i.e., the region where the parameter $t$ decreases upon increasing density.

diagram shrinks and it goes to lower pressures. In the case in which $b=-1.00$, shown in Fig. 6(e), the diffusion constant behaves as in a normal liquid. The diffusion anomalous region lies, as one would expect, in the low density liquid phase where particles are less bound. As the attractive part of the potential becomes deeper, particles become more bound, favoring the high density liquid phase, and eliminating the possibility of anomalous mobility.

\section{Structural anomaly}

Besides the density and the diffusion anomalies, a structural anomalous region might be present. Figures 7(a)-7(e) show the translational order parameter defined by Eq. (6) as a function of density for fixed temperatures for the potential we are studying for $b=0.0,-0.25,-0.50,-0.75$, and -1.00 . 

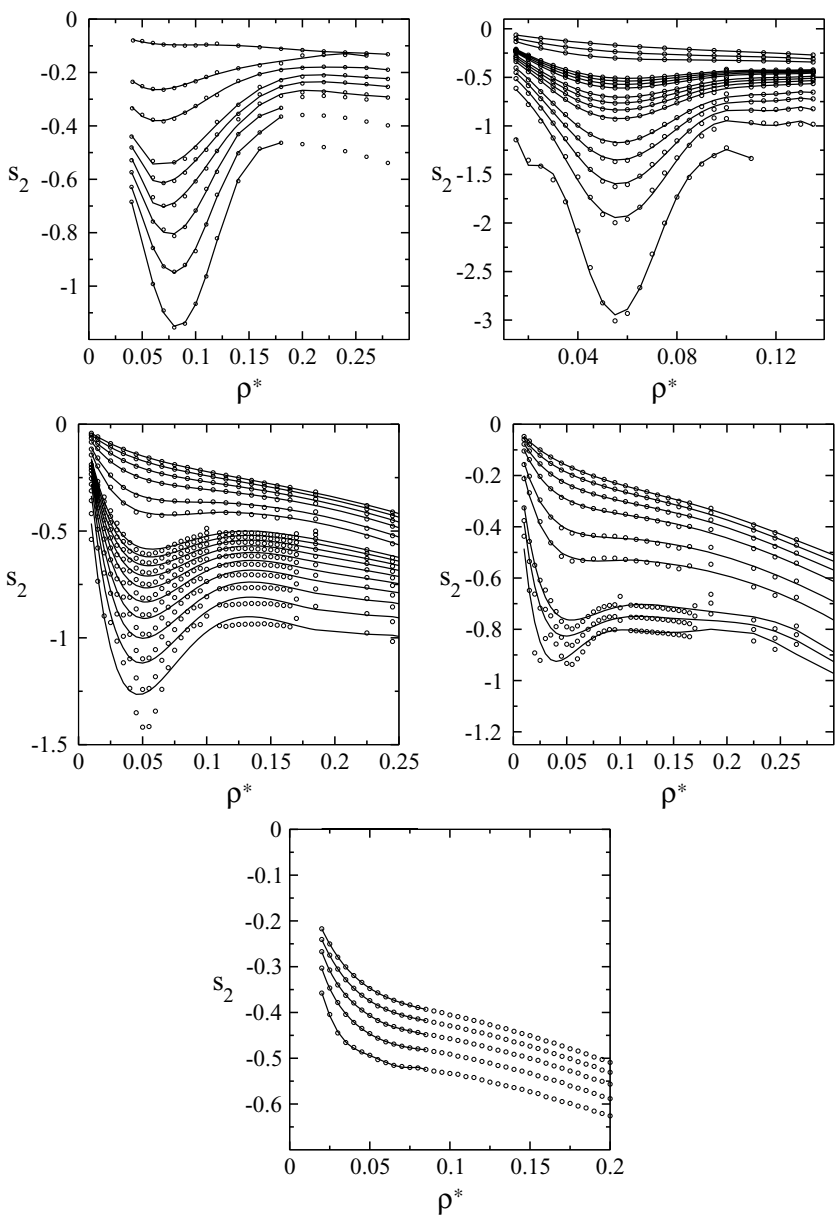

FIG. 8. Excess entropy vs density for:(a) $b=0.00$ and $T^{*}=0.25,0.30, \ldots$, $0.7,1.0,3.0$, and 5.0 ; (b) $b=-0.25$ and $T^{*}=0.08,0.10, \ldots, 0.18,0.24$, $0.26,0.28,0.32,0.36,0.44,0.48,0.52,0.54,0.56,0.64,0.68,0.72,1.20$, 1.60 , and 2.50 ; (c) $b=-0.50$ and $T^{*}=0.08,0.10, \ldots, 0.18,0.24,0.26$, $0.28,0.32,0.36,0.44,0.48,0.52,0.54,0.56,0.64,0.68,0.72,0.76,0.80$, $1.00,1.20,1.60,2.00,2.50$, and 3.00 ; (d) $b=-0.75$ and $T^{*}=0.24,0.26$, $0.28,0.32,0.36,0.40,0.44, \ldots, 0.80,1.00,1.20,1.60,2.00,2.50$, and 3.00 ; (e) $b=-1.00$ and $T^{*}=1.10,1.15,1.20,1.30, \ldots, 1.70,2.00$, and 3.00 .

The dots represent the simulation data and the solid lines are polynomial fit to the data.

The nonmonotonic behavior of these curves indicates that there is a region in which $t$ decreases with density. This means that the system becomes less structured for increasing density. Dotted lines determine the local maxima and minima of $t$, bounding the structural anomalous region. This region was mapped into the pressure-temperature phase diagram (dotted lines), as can be seen in Figs. 2(a)-2(e). The comparison between the behavior for different $b$ values indicates that as the attractive well becomes deeper the structural anomalous region in the pressure-temperature phase diagram shrinks and moves to lower pressures and it is still present even in the deepest case, $b=-1.00$. According to these results, we believe that for $b<-1.00$, i.e., cases in which the attractive part is more intense than one showed in case $\mathrm{E}$, the structural anomalous region will also vanish. This result again is consistent with the idea that a deeper attractive term favors the high density liquid phase.
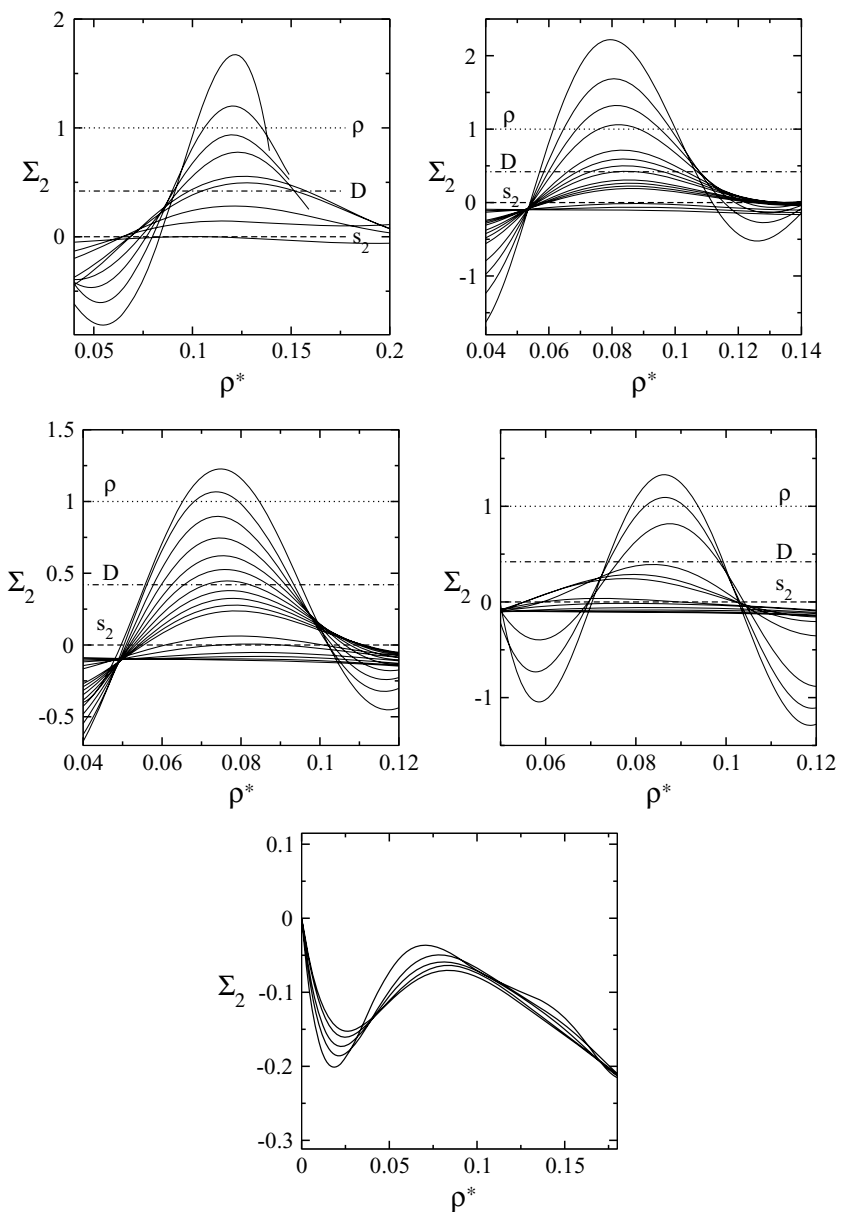

FIG. 9. Derivative of the excess entropy with respect to density vs density for: (a) $b=0.00$ and $T^{*}=0.25,0.30, \ldots, 0.5,0.7,1.0,3.0$, and 5.0 ; (b) $b=-0.25$ and $T^{*}=0.08,0.10, \ldots, 0.18,0.20,0.24,0.28,0.32$, $0.36,0.44,0.48,0.52,0.54,0.56,0.64,0.68,0.72,1.20,1.60$, and 2.50 ; (c) $b=-0.50$ and $T^{*}=0.08,0.10, \ldots, 0.18,0.24,0.26,0.28,0.32,0.36$, $0.44,0.48,0.52,0.54,0.56,0.64,0.68,0.72,0.76,0.80,1.00,1.20,1.60,2.00$, 2.50 , and 3.00 ; (d) $b=-0.75$ and $T^{*}=0.24,0.26,0.28,0.32,0.36,0.40$, $0.44,0.48,0.52,0.56,0.60,0.64,0.68,0.72,0.76,0.80,1.00,1.20,1.60$, $2.00,2.50$, and 3.00; (e) $b=-1.00$ and $T^{*}=1.10,1.15,1.20,1.30,1.40$, $1.50,1.60,1.70,2.00$, and 3.00 .

Complementary to the structural anomalous region obtained by analyzing the parameter $t$, we can gain some understanding about the presence or absence of these anomalies and the shape of the potential by analyzing the density dependence of the excess entropy. ${ }^{67}$ The excess entropy is defined as the difference between the entropy of the real fluid and that of an ideal gas at the same temperature and density. Errington $e t$ al. have shown that the density anomaly is given by the condition $\Sigma_{\mathrm{ex}}=\left(\partial s_{\mathrm{ex}} / \partial \ln \rho\right)_{T}>1 .{ }^{67}$ They have also shown that the two body contribution of $s_{\mathrm{ex}}$,

$$
s_{\mathrm{ex}} \approx s_{2}=-2 \pi \rho \int[g(r) \ln g(r)-g(r)+1] r^{2} d r,
$$

gives a good approximation of $s_{\mathrm{ex}}$. The radial distribution function, $g(r)$, is proportional to the probability to find a particle at a distance $r$ to another particle placed at the origin. Errington et al. ${ }^{67}$ have also suggested that the diffusion anomaly can be predicted by using the empirical Rosenfeld's parametrization. ${ }^{68}$ They found the condition $\Sigma_{2}>0.42$ for a 

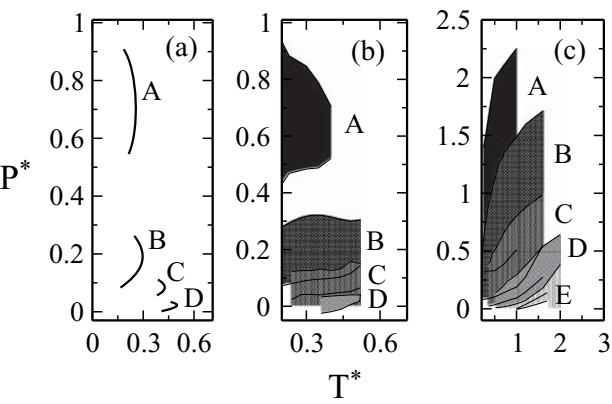

FIG. 10. (a) $T M D$ line for the cases considered in this paper. Note that there is no density anomaly in the case E [see Figs. 2(a)-2(e)]. (b) The diffusion anomaly region for the cases A-D. No diffusion anomaly was found for the case E [see Figs. 6(a)-6(e)]. The shadowed regions correspond to the region between the dashed lines in Figs. 2(a)-2(e). In (c) is shown the structural anomalous region for cases A-E. Here, the shadowed region corresponds to the region between the dotted lines in Figs. 2(a)-2(e). All these results were obtained by $N V T$ simulations.

diffusion anomalous behavior. They also claim that $\Sigma_{2}>0$ is a good estimate for determining the region where structural anomaly occurs. ${ }^{67}$

Here we test these assumptions in our potential for the different depth of the attractive term.

Figures 8(a)-8(e) illustrate the excess entropy versus density for the cases $b=0,-0.25,-0.50,-0.75$ and $b=1.0$ for various temperatures. For the case $b$ $=0,-0.25,-0.50,-0.75$, the excess entropy as a function of density has a range of temperatures and densities where the excess entropy has positive slope.

Figures 9(a)-9(e) illustrate the derivative of the excess entropy with respect to the density versus density for the cases $b=0,-0.25,-0.50,-0.75$ and $b=1.0$ for various temperatures. For the cases $b=0,-0.25,-0.50,-0.75$ the derivative of the excess entropy as a function of density has a range of temperatures and densities where $\Sigma_{2}>1$, $\Sigma_{2}>0.42, \Sigma_{2}>0$ what indicates the presence of density, diffusion, and structural anomalous regions. This is not the case for $b=1.0$.

Figure 10 gives an overview of the density, diffusion, and structural anomaly locations in the pressure-temperature phase diagram.

\section{CONCLUSIONS}

In this paper, we have explored the effect of the addition of an attractive part in a two length scale potential. Particularly we analyze if the depth of the attractive part changes the position (and the presence or not) of the two liquid-gas and liquid-liquid critical points and of the density, diffusion, and structural anomalous regions in the pressure-temperature phase diagram.

Our results show that the depth of attractive part of pair interaction potential is directly related with the low density liquid. Analyzing the evolution of liquid-gas critical point at $P T$ phase diagram, we noted that it moves out to higher temperatures as the attractive part of pair potential increase. The increase of the deep brings stability to the low density liquid phase. Thus, more energy is necessary to push out the system from this state. When this energy is given through an increase of temperature, the system goes to the gas phase.

The main effect of the pressure is to keep particles close to the equilibrium point of the repulsive part. This makes it possible for the particles to move from the attractive part to the repulsive part of the potential. As the well becomes deeper, particles are trapped in the minimum of the attractive part. Hence a high pressure is not necessary to keep particles closer to the repulsive shoulder.

For sufficiently intense attraction between particles, both the liquid-liquid and the liquid-gas critical points are present. These two critical points are observed even for a very attractive potential. For a small attractive interaction, only the liquid-gas critical point was found what indicates that for the coexistence of two liquid phases the attractive well have to be deeper than a certain threshold.

Since the attraction favors the liquid phase (particularly the high density liquid phase), as the $b$ decreases the liquidgas critical point moves to higher temperatures (shown in Fig. 4) and the liquid-liquid critical point to lower pressures.

The density, diffusion, and structural anomalous regions are present even in the absence of attraction. As $b$ decreases, the high density liquid structure is favored and so the anomalous regions in the pressure-temperature phase diagram (shown in Fig. 10) shrinks, moves to lower pressures, and disappears for very attractive potentials. The analysis of the excess entropy with density supports these results.

In order to resume, the density and the diffusion anomalies are present in systems which interact through two length scale potentials if the particle move from one scale to the other. If the attractive scale is too deep, the flux of particles between the scales decreases and no anomalies are observed.

\section{ACKNOWLEDGMENTS}

We thank for financial support from the Brazilian science agencies CNPq, CAPES and FAPEMIG. This work is also partially supported by the CNPq through the INCT-FCx.

${ }^{1}$ Periodic table of the elements, http://periodic.lanl.gov/default.htm, 2007.

${ }^{2}$ R. Waler, Essays of Natural Experiments (Johnson Reprint, New York, 1964).

${ }^{3}$ F. X. Prielmeier, E. W. Lang, R. J. Speedy, and H.-D. Lüdemann, Phys. Rev. Lett. 59, 1128 (1987).

${ }^{4}$ L. Haar, J. S. Gallangher, and G. Kell, NBS/NRC Steam Tables. Thermodynamic and Transport Properties and Computer Programs for Vapor and Liquid States of Water in SI Units, 1st ed. (Hemisphere, Washington, DC, 1984).

${ }^{5}$ C. A. Angell, E. D. Finch, and P. Bach, J. Chem. Phys. 65, 3063 (1976).

${ }^{6}$ P. A. Netz, F. W. Starr, H. E. Stanley, and M. C. Barbosa, J. Chem. Phys. 115, 344 (2001).

${ }^{7}$ P. A. Netz, F. W. Starr, M. C. Barbosa, and H. E. Stanley, Journal of Molecular Liquids 101, 159 (2002).

${ }^{8}$ J. R. Errington and P. G. Debenedetti, Nature (London) 409, 318 (2001).

${ }^{9}$ J. Mittal, J. R. Errington, and T. M. Truskett, J. Phys. Chem. B 110, 18147 (2006).

${ }^{10}$ A. Mudi, C. Chakravarty, and R. Ramaswamy, J. Chem. Phys. 122, 104507 (2005).

${ }^{11}$ H. J. C. Berendsen, J. R. Grigera, and T. P. Straatsma, J. Phys. Chem. 91, 6269 (1987).

${ }^{12}$ O. Mishima, J. Chem. Phys. 100, 5910 (1994).

${ }^{13}$ O. Mishima and H. E. Stanley, Nature (London) 396, 329 (1998).

${ }^{14}$ P. H. Poole, F. Sciortino, U. Essmann, and H. E. Stanley, Nature (London) 360, 324 (1992). 
${ }^{15}$ P. A. Netz, F. W. Starr, M. C. Barbosa, and H. E. Stanley, Physica A 314, 470 (2002).

${ }^{16}$ O. Mishima and H. E. Stanley, Nature 392, 164 (1998).

${ }^{17}$ H. Thurn and J. Ruska, J. Non-Cryst. Solids 22, 331 (1976).

${ }^{18}$ G. E. Sauer and L. B. Borst, Science 158, 1567 (1967).

${ }^{19}$ S. J. Kennedy and J. C. Wheeler, J. Chem. Phys. 78, 1523 (1983).

${ }^{20}$ T. Tsuchiya, J. Phys. Soc. Jpn. 60, 227 (1991).

${ }^{21}$ P. T. Cummings and G. Stell, Mol. Phys. 43, 1267 (1981).

${ }^{22}$ M. Togaya, Phys. Rev. Lett. 79, 2474 (1997).

${ }^{23}$ C. A. Angell, R. D. Bressel, M. Hemmatti, E. J. Sare, and J. C. Tucker, Phys. Chem. Chem. Phys. 2, 1559 (2000).

${ }^{24}$ R. Sharma, S. N. Chakraborty, and C. Chakravarty, J. Chem. Phys. 125, 204501 (2006).

${ }^{25}$ M. S. Shell, P. G. Debenedetti, and A. Z. Panagiotopoulos, Phys. Rev. E 66, 011202 (2002).

${ }^{26}$ S. Sastry and C. A. Angell, Nature Mater. 2, 739 (2003).

${ }^{27}$ S. H. Chen, F. Mallamace, C. Y. Mou, M. Broccio, C. Corsaro, A. Faraone, and L. Liu, Proceedings of the National Acad. Sci. U.S.A. 103, 12974 (2006).

${ }^{28}$ T. Morishita, Phys. Rev. E 72, 021201 (2005).

${ }^{29}$ S. V. Buldyrev and H. E. Stanley, Physica A 330, 124 (2003).

${ }^{30}$ A. Skibinsky, S. V. Buldyrev, G. Franzese, G. Malescio, and H. E. Stanley, Phys. Rev. E 69, 061206 (2005).

${ }^{31}$ V. B. Henriques, N. Guissoni, M. A. Barbosa, M. Thielo, and M. C. Barbosa, Mol. Phys. 103, 3001 (2005).

${ }^{32}$ P. C. Hemmer and G. Stell, Phys. Rev. Lett. 24, 1284 (1970).

${ }^{33}$ E. A. Jagla, Phys. Rev. E 58, 1478 (1998).

${ }^{34}$ N. B. Wilding and J. E. Magee, Phys. Rev. E 66, 031509 (2002).

${ }^{35}$ A. B. de Oliveira, P. A. Netz, T. Colla, and M. C. Barbosa, J. Chem. Phys. 124, 084505 (2006).

${ }^{36}$ N. G. Almarza, J. A. Capitan, J. A. Cuesta, and E. Lomba, J. Chem. Phys. 131, 124506 (2009).

${ }^{37}$ D. Y. Fomin, N. V. Gribova, V. N. Ryzhov, S. M. Stishov, and D. Frenkel, J. Chem. Phys. 129, 064512 (2008).

${ }^{38}$ G. Franzese, J. Mol. Liq. 136, 267 (2007).

${ }^{39}$ A. B. de Oliveira, G. Franzese, P. A. Netz, and M. C. Barbosa, J. Chem. Phys. 128, 064901 (2008).

${ }^{40}$ A. B. de Oliveira, P. A. Netz, and M. C. Barbosa, Europhys. Lett. 85, 36001 (2009).

${ }^{41}$ S. Zhou, Phys. Rev. E 74, 031119 (2006).

${ }^{42}$ S. Zhou, Phys. Rev. E 77, 041110 (2008).

${ }^{43}$ S. Zhou, J. Chem. Phys. 130, 054103 (2009).

${ }^{44}$ S. A. Egorov, J. Chem. Phys. 128, 174503 (2008).

${ }^{45}$ N. V. Gribova, Y. D. Fomin, D. Frenkel, and V. N. Ryzhov, Phys. Rev. E 79, 051202 (2009).
${ }^{46}$ E. G. Noya, C. Vega, J. P. K. Doye, and A. A. Louis, J. Chem. Phys. 127, 054501 (2007).

${ }^{47}$ S. V. Buldyrev, G. Malescio, C. A. Angell, N. Giovambattista, S. Prestipino, F. Saija, H. E. Stanley, and L. Xu, J. Phys.: Condens. Matter 21, 504106 (2009).

${ }^{48}$ E. Lomba, N. G. Almarza, C. Martin, and C. McBride, J. Chem. Phys. 126, 244510 (2007).

${ }^{49}$ A. B. de Oliveira, P. A. Netz, T. Colla, and M. C. Barbosa, J. Chem. Phys. 125, 124503 (2006).

${ }^{50}$ A. B. de Oliveira, M. C. Barbosa, and P. A. Netz, Physica A 386, 744 (2007).

${ }^{51}$ A. B. de Oliveira, P. A. Netz, and M. C. Barbosa, European Physical Jouranl B 64, 481 (2008).

${ }^{52}$ A. B. de Oliveira, E. Salcedo, C. Chakravarty, and M. C. Barbosa, J. Chem. Phys. 132, 234509 (2010).

${ }^{53}$ A. B. de Oliveira, E. Salcedo, C. Chakravarty, and M. C. Barbosa, J. Chem. Phys. 132, 234509 (2010).

${ }^{54}$ W. P. Krekelberg, J. Mittal, V. Ganesan, and T. M. Truskett, Phys. Rev. E 77, 041201 (2008).

${ }^{55}$ Z. Yan, S. V. Buldyrev, P. Kumar, N. Giovambattista, P. G. Debenedetti, and H. E. Stanley, Phys. Rev. E 76, 051201 (2007).

${ }^{56}$ C. H. Cho, S. Singh, and G. W. Robinson, Faraday Discuss. 103, 19 (1996).

${ }^{57}$ P. A. Netz, J. F. Raymundi, A. S. Camera, and M. C. Barbosa, Physica A 342, 48 (2004).

${ }^{58}$ S. J. Plimpton, J. Comp. Phys. 117, 1 (1995).

${ }^{59}$ T. M. Truskett, S. Torquato, and P. G. Debenedetti, Phys. Rev. E 62, 993 (2000).

${ }^{60}$ J. E. Errington, P. G. Debenedetti, and S. Torquato, J. Chem. Phys. 118, 2256 (2003).

${ }^{61}$ D. Frenkel and B. Smit, Understanding Molecular Simulation, 1st ed. (Academic, San Diego, 1996).

${ }^{62}$ G. Malescio, G. Franzese, G. Pellicane, A. Skibinsky, S. V. Buldyrev, and H. E. Stanley, J. Phys.: Condens. Matter 14, 2193 (2002).

${ }^{63}$ G. Malescio, G. Franzese, A. Skibinsky, S. V. Buldyrev, and H. E. Stanley, Phys. Rev. E 71, 061504 (2005).

${ }^{64}$ H. E. Stanley, S. V. Buldyrev, M. Canpolat, M. Meyer, O. Mishima, M. R. Sadr-Lahijany, A. Scala, and F. W. Starr, Physica A 257, 213 (1998).

${ }^{65}$ H. E. Stanley, Pramana: Journal of Physica 53, 53 (1999).

${ }^{66}$ H. E. Stanley, S. V. Buldyrev, M. Canpolat, O. Mishima, A. SadrLahijany, M. R. Scala, and F. W. Starr, Phys. Chem. Chem. Phys. 2, 1551 (2000).

${ }^{67}$ J. R. Errington, T. M. Truskett, and J. Mittal, J. Chem. Phys. 125, 244502 (2006).

${ }^{68}$ Y. Rosenfeld, J. Phys.: Condens. Matter 11, 5415 (1999). 